\title{
Combining clinicopathological predictors and molecular biomarkers in the oncogenic K-RAS/Ki67/HIF-1 $\alpha$ pathway to predict survival in resectable pancreatic cancer
}

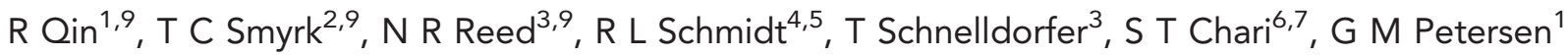 \\ and A H Tang ${ }^{\star 3,4,8}$ \\ ${ }^{1}$ Department of Health Sciences Research, Mayo Clinic Cancer Center, Mayo Clinic, Rochester, MN 55905, USA; ${ }^{2}$ Division of \\ Anatomic Pathology, Department of Laboratory Medicine and Pathology, Mayo Clinic Cancer Center, Mayo Clinic, Rochester, \\ MN 55905, USA; ${ }^{3}$ Department of Surgery, Mayo Clinic Cancer Center, Mayo Clinic, Rochester, MN 55905, USA; ${ }^{4}$ Department of \\ Biochemistry and Molecular Biology, Mayo Clinic Cancer Center, Mayo Clinic, Rochester, MN 55905, USA; ${ }^{5}$ Upper lowa University, \\ Fayette, IA 52142, USA; ${ }^{6}$ Department of Medicine, Mayo Clinic Cancer Center, Mayo Clinic, Rochester, MN 55905, USA; \\ ${ }^{7}$ Department of Oncology, Mayo Clinic Cancer Center, Mayo Clinic, Rochester, MN 55905, USA and ${ }^{8}$ Department of Microbiology \\ and Molecular Cell Biology, Leroy T. Canoles Jr. Cancer Research Center, Eastern Virginia Medical School, Norfolk, VA 23507, USA
}

Background: The dismal prognosis of patients diagnosed with pancreatic cancer points to our limited arsenal of effective anticancer therapies. Oncogenic K-RAS hyperactivation is virtually universal in pancreatic cancer, that confers drug resistance, drives aggressive tumorigenesis and rapid metastasis. Pancreatic tumours are often marked by hypovascularity, increased hypoxia and ineffective drug delivery. Thus, biomarker discovery and developing innovative means of countervailing oncogenic K-RAS activation are urgently needed.

Methods: Tumour specimens from 147 pancreatic cancer patients were analysed by immunohistochemical (IHC) staining and tissue microarray (TMA). Statistical correlations between selected biomarkers and clinicopathological predictors were examined to predict survival.

Results: We find that heightened hypoxia response predicts poor clinical outcome in resectable pancreatic cancer. SIAH is a tumour-specific biomarker. The combination of five biomarkers (EGFR, phospho-ERK, SIAH, Ki67 and HIF-1 $\alpha$ ) and four clinicopathological predictors (tumour size, pathological grade, margin and lymph node status) predict patient survival post surgery in pancreatic cancer.

Conclusions: Combining five biomarkers in the K-RAS/Ki67/HIF-1 $\alpha$ pathways with four clinicopathological predictors may assist to better predict survival in resectable pancreatic cancer.

Pancreatic cancer is the fourth leading cause of cancer-related death in the United States with a dismal 5-year survival rate at 5-6\% (Siegel et al, 2013). Clinical symptoms associated with pancreatic ductal adenocarcinomas (PDAC) manifest by late diagnosis, local invasion and systemic metastasis-culminating in dismal prognosis and poor survival (Wolfgang et al, 2013). Surgical

\footnotetext{
*Correspondence: Professor A Tang; E-mail: TangAH@evms.edu

${ }^{9}$ These authors share co-1st authorship with equal contribution.
}

Revised 25 November 2014; accepted 10 December 2014; published online 13 January 2015

(C) 2015 Cancer Research UK. All rights reserved 0007-0920/15 
resection offers the best hope for increased survival if the disease is discovered before local and distant metastasis (Sohn et al, 2000). However, a minority of patients $(\sim 15 \%)$ are eligible for surgical resection at the time of diagnosis (Hidalgo, 2010). Even those with resectable tumours frequently experience post-surgery relapse, succumbing to this deadly malignancy (Katz et al, 2009). Adjuvant therapies confer limited or modest benefit (Conroy et al, 2011; Valle et al, 2014). As a result, there is an urgent need to discover new biomarkers aiding in prognosis and to develop curative strategies against pancreatic cancer (Maitra and Hruban, 2008).

The cancer genome-sequencing project reaffirmed the paramount importance of oncogenic K-RAS activation in human cancer (Vogelstein et al, 2013). The mitogen-activated protein kinase (MAPK/ERK) cascade is often activated in human cancer (Sebolt-Leopold and Herrera, 2004). Previous studies suggested that upregulation in phospho-ERK (that is, active ERK) is associated with reduced survival in pancreatic cancer (Javle et al, 2007). Ectopic expression of EGFR in pancreatic cancer has been linked to accelerated tumour growth, increased metastasis and poor survival (Ardito et al, 2012). Pancreatic cancer is well known to be desmoplastic, hypovascularised and treatment-resistant (Von Hoff et al, 2009), and increased expression of hypoxiainducible factor $1 \alpha$ (HIF- $1 \alpha)$ may alter cellular metabolism, aid tumour growth and increase cancer survival in the hypoxic and nutrient-deprived tumour environment (Akakura et al, 2001; Spivak-Kroizman et al, 2013).

Many genes have been evaluated as prognostic biomarkers for pancreatic cancer, but most were unreliable and inconsistent in clinical validations (Sawyers, 2008; Costello et al, 2012; Winter et al, 2013). As tumorigenesis requires multiple molecular alterations to progress, a logical combination of several biomarkers in the main tumour-promoting signalling pathways may improve the prognostic accuracy. Here, we examined the prognostic value of six biomarkers: upstream receptors (EGFR and HER2), midstream kinase (phospho-ERK) and K-RAS downstream signalling 'gatekeeper' (SIAH E3 ligase; Schmidt et al, 2007; Ahmed et al, 2008), tumour proliferation index (Ki67) and hypoxic response indicator $($ HIF- $1 \alpha)$ in human pancreatic cancer. Along with four clinicopathological predictors, we focused on the oncogenic K-RAS/Ki67/ HIF- $1 \alpha$ pathways by evaluating the prognostic value of these six aforementioned biomarkers. SIAH is a new tumour-specific biomarker in pancreatic cancer. We report that combining the five biomarkers (EGFR, phospho-ERK, SIAH, HIF-1 $\alpha$ and Ki67) together with the four existing clinicopathological predictors (tumour size, pathological grade, margin and lymph node status) is clearly predictive for patient survival. Importantly, an increased expression of HIF- $1 \alpha$ predict poor prognosis in pancreatic cancer.

\section{MATERIALS AND METHODS}

Ethical statement. This study was conducted with the ethical committee approval by the Mayo Clinic Institutional Review Board. Informed consent was obtained from pancreatic cancer patients before their surgeries at the Mayo Clinic.

Patients. Patients who underwent surgical resection for PDAC at the Mayo Clinic in Rochester, MN between 1985 and 2001 were included in this study $(n=147)$. Patients underwent either pancreaticoduodenectomy $(n=129)$, distal pancreatectomy $(n=8)$ or total pancreatectomy $(n=10)$. Clinical and pathologic variables were extracted from the patients' medical records (Table 1). Nineteen patients $(12.9 \%)$ were lost to follow-up; survival times were censored at the time of last known contact (2007).

Pathologic samples/tissue microarray (TMA). Biospecimens from 134 pancreatic cancer patients were used to construct this TMA. Owing to tumour heterogeneity, each patient's tumour specimen was represented by three distinct, pathologically confirmed tumour cores (diameter $=0.6 \mathrm{~mm}$ ) on the TMA. The numbers of TMA tissue cores and patients used in the statistical analyses are summarised (Table 2). Tumour biospecimens from 13 pancreatic cancer patients at distinct pathological stages (Hruban et al, 2000) were used to validate the TMA results. Normal pancreas and pancreatitis tissues were served as negative controls. All tissue cores and histology sections were carefully reviewed by an expert pathologist in a double blind manner and classified into normal pancreas $(n=7)$, pancreatitis $(n=20)$, PanIN-1 $(n=35)$, PanIN-2 $(n=20)$, PanIN-3 $(n=15)$, and adenocarcinoma ADCA $2(n=84)$, ADCA $3(n=161)$ and ADCA $4(n=33)$.

Five-micrometre sections were cut from each TMA slide and deparaffinised using standard techniques, and then placed in $1.0 \mathrm{~mm}$ EDTA (pH 8.0) for $30 \mathrm{~min}$ at $100^{\circ} \mathrm{C}$ for antigen retrieval. Staining was conducted using monoclonal anti-EGFR antibody (pre-diluted, Dako, Glostrup, Denmark), monoclonal anti-phospho-ERK (1:750 dilution, Cell Signaling, Danvers, MA, USA), monoclonal anti-HER2 (pre-diluted, Dako), monoclonal anti-HIF-1 $\alpha$ (1:250 dilution, Novus Biologicals, Littleton, CO, USA), monoclonal anti-Ki67 ( $1: 100$ dilution, Dako) and monoclonal anti-SIAH antibodies $(1: 40$ dilution, Novus Biologicals) (Schmidt et al, 2007). A board-certified pathologist, with 32 years of experience in pancreatic cancer, scored and reviewed TMA slides and immunohistochemical (IHC) staining. Percent staining scores were measured for each core. Scores were averaged across three tissue cores from the same tumour to yield a single percent staining score representing each cancer patient. All IHC images were captured $(\times 400)$ using Leica compound microscope and DC500 camera.

Statistical analysis. Descriptive statistics including the mean (s.d.), median (range) and frequency summarise the data (Table 1). Spearman's correlation coefficients were computed to explore the correlations among clinicopathological variables and biomarker variables (Table 3). Survival time was calculated in days from the date of surgery to the date of death, or censored at the date of final contact as of 1 October 2007 if patients were lost to follow-up. Survival curves were analysed by the Kaplan-Meier method. In univariate analysis, the log-rank test was used to calculate the difference in survival for categorical variables, whereas the Cox proportional hazards $(\mathrm{PH})$ model was used to examine the effect on survival for continuous variables. Multivariate survival analysis was carried out using the Cox PH models after controlling for the four clinicopathological predictors. The $\mathrm{PH}$ assumptions were verified by using the log-transformation plot and the goodness-of-fit of the models were checked by martingale and deviance residual plots. The time-dependent receiver operating characteristic (survival ROC) was applied to display and compare the sensitivity and specificity of the predictive models based on the multivariate survival analysis (Heagerty and Zheng, 2005). The survival ROC curves were used to accommodate the timedependent nature and censoring in the survival data. All $P$-values were two-sided. A $P$-value of less than 0.05 was considered statistically significant. The statistical plots were generated using the SAS software Version 9.1.3 (SAS Institute Inc, Cary, NC, USA) and $\mathrm{R}$ software Version 2.6.1 (R Foundation for Statistical Computing, Vienna, Austria).

\section{RESULTS}

SIAH specifically decorates proliferating tumour cells in human pancreatic cancer. As the most downstream signalling 'gatekeeper' identified in the K-RAS pathway (Figure 1A), SIAH is a logical and potent anti-K-RAS drug target in cancer (Schmidt et al, 2007; Ahmed et al, 2008). SIAH-positive cells in pancreatic tumours are of epithelial origin in adult pancreas, and SIAH has a 


\section{Table 1. Demographic, clinical and biomarker variables for pancreatic cancer-patient survival}

\begin{tabular}{|c|c|c|c|c|}
\hline Variables & Level & Mean/frequency & s.d./(\%) & $P$-value ${ }^{a}$ \\
\hline Age at surgery (year) & Mean & 63.1 & 12.0 & 0.077 \\
\hline Gender & $\begin{array}{l}\text { Male } \\
\text { Female }\end{array}$ & $\begin{array}{l}80 \\
67\end{array}$ & $\begin{array}{l}54.4 \% \\
45.6 \%\end{array}$ & 0.530 \\
\hline Surgical procedure & $\begin{array}{l}\text { Standard pancreaticoduodenectomy } \\
\text { Pylorus-preserving pancreaticoduodenectomy } \\
\text { Distal pancreatectomy } \\
\text { Total pancreatectomy }\end{array}$ & $\begin{array}{c}77 \\
52 \\
8 \\
10\end{array}$ & $\begin{array}{l}52.4 \% \\
34.4 \% \\
5.4 \% \\
6.8 \%\end{array}$ & 0.542 \\
\hline Complete resection & $\begin{array}{l}\text { Missing } \\
\text { No } \\
\text { Yes }\end{array}$ & $\begin{array}{c}1 \\
19 \\
127\end{array}$ & $\begin{array}{l}13 \% \\
87 \%\end{array}$ & 0.584 \\
\hline Tumour size & $\begin{array}{l}\text { Mean } \\
\leqslant 2 \mathrm{~cm} \\
>2 \mathrm{~cm}\end{array}$ & $\begin{array}{c}2.9 \\
44 \\
103\end{array}$ & $\begin{array}{c}1.15 \\
20.9 \% \\
70.1 \%\end{array}$ & 0.164 \\
\hline Lymph node status & $\begin{array}{l}N_{0} \\
N_{1}\end{array}$ & $\begin{array}{l}74 \\
73\end{array}$ & $\begin{array}{l}50.3 \% \\
49.7 \%\end{array}$ & 0.343 \\
\hline Positive margin & $\begin{array}{l}\mathrm{R}_{0} \\
\mathrm{R}_{1} / \mathrm{R}_{2}\end{array}$ & $\begin{array}{c}127 \\
20\end{array}$ & $\begin{array}{l}86.4 \% \\
13.6 \%\end{array}$ & 0.461 \\
\hline UICC stage & $\begin{array}{l}\text { Stage IA } \\
\text { Stage IB } \\
\text { Stage IIA } \\
\text { Stage IIB/IV }\end{array}$ & $\begin{array}{l}12 \\
18 \\
43 \\
74\end{array}$ & $\begin{array}{l}8.2 \% \\
12.2 \% \\
29.2 \% \\
50.4 \%\end{array}$ & 0.703 \\
\hline Tumour grade & $\begin{array}{l}2 \\
3 \\
4\end{array}$ & $\begin{array}{l}38 \\
73 \\
36\end{array}$ & $\begin{array}{l}25.9 \% \\
49.7 \% \\
24.5 \%\end{array}$ & $<0.001$ \\
\hline Extension & $\begin{array}{l}\text { No } \\
\text { Yes }\end{array}$ & $\begin{array}{l}58 \\
89\end{array}$ & $\begin{array}{l}39.5 \% \\
60.5 \%\end{array}$ & 0.284 \\
\hline Perineural invasion & $\begin{array}{l}\text { Missing } \\
\text { No } \\
\text { Yes }\end{array}$ & $\begin{array}{l}18 \\
74 \\
55\end{array}$ & $\begin{array}{l}57.4 \% \\
42.6 \%\end{array}$ & 0.696 \\
\hline Angiolymphatic invasion & $\begin{array}{l}\text { Missing } \\
\text { No } \\
\text { Yes }\end{array}$ & $\begin{array}{l}18 \\
98 \\
31\end{array}$ & $\begin{array}{l}76.0 \% \\
24.0 \%\end{array}$ & 0.253 \\
\hline Adjuvant therapy (chemotherapy and radiation therapy) & $\begin{array}{l}\text { Missing } \\
\text { No } \\
\text { Yes }\end{array}$ & $\begin{array}{c}7 \\
26 \\
115\end{array}$ & $\begin{array}{l}18.4 \% \\
81.6 \%\end{array}$ & 0.883 \\
\hline $\begin{array}{l}\text { Biomarkers } \\
\text { EGFR } \\
\text { SIAH } \\
\text { Ki67 } \\
\text { Phospho-ERK } \\
\text { HIF-1 } \alpha\end{array}$ & $\begin{array}{l}\text { Number of patients } \\
123 \\
127 \\
119 \\
131 \\
100\end{array}$ & $\begin{array}{c}2.79 \\
42.30 \\
39.33 \\
36.22 \\
38.27\end{array}$ & $\begin{array}{c}1.52 \\
22.11 \\
24.22 \\
28.14 \\
33.12\end{array}$ & $\begin{array}{l}0.556 \\
0.235 \\
0.171 \\
0.185 \\
0.005\end{array}$ \\
\hline Survival & Median time (days) & 541 & $\begin{array}{l}(460- \\
615)\end{array}$ & - \\
\hline \multicolumn{5}{|l|}{ Vital status } \\
\hline & $\begin{array}{l}\text { Alive } \\
\text { Dead }\end{array}$ & $\begin{array}{c}19 \\
128\end{array}$ & $\begin{array}{l}12.9 \% \\
87.1 \%\end{array}$ & \\
\hline
\end{tabular}

predominantly nuclear expression pattern, highly specific to tumour cells (Figure 1B and D). SIAH staining was not detected in the normal pancreas, pancreatitis tissues, tumour stroma and infiltrating immune cells (Figure 1D and data not shown). Numbers of SIAH-positive tumour cells increase as human pancreatic tumours progress through well-defined neoplastic stages-PanIN-1, PanIN-2, PanIN-3 and ADCAs (Figure 1B, D and $2 \mathrm{~B}$ ), correlating SIAH expression with active tumour proliferation. Therefore, SIAH-dependent proteolysis in proliferating tumour cells may facilitate oncogenic K-RAS signalling, 
Table 2. IHC staining of six biomarkers in the K-RAS/Ki67/HIF-1 $\alpha$ pathways

\begin{tabular}{|l|c|c|c|c|c|}
\hline Biomarker & Total samples stained & Positive samples & Total patients stained & Positive patients & $\begin{array}{c}\text { Median expression, } \\
\text { range of positive samples }\end{array}$ \\
\hline EGFR & 249 & $192(77 \%)$ & 110 & $94(85 \%)$ & $2(0-4)$ \\
\hline HER2 & 287 & $56(19 \%)$ & 117 & $32(27 \%)$ & $0(0-2)$ \\
\hline PERK & 301 & $219(72 \%)$ & 131 & $106(81 \%)$ & $40 \%(5-90 \%)$ \\
\hline SIAH & 315 & $303(96 \%)$ & 127 & $127(100 \%)$ & $30 \%(5-90 \%)$ \\
\hline Ki67 & 250 & $237(95 \%)$ & 119 & $118(99 \%)$ & $40 \%(5-90 \%)$ \\
\hline HIF-1- $\alpha$ & 210 & $143(69 \%)$ & 100 & $77(77 \%)$ & $40 \%(2-90 \%)$ \\
\hline Abbreviations: HIF-1 $\alpha=$ hypoxia-inducible factor $1 \alpha ; \mathrm{HC}=$ immunohistochemical. \\
\hline
\end{tabular}

Table 3. Spearman's correlation coefficients between four clinical variables and five biomarkers

\begin{tabular}{|c|c|c|c|c|c|c|c|c|c|}
\hline Biomarker & Lymph node & Pathological grade & Extension & Tumour size & EGFR & SIAH & Ki67 & Phospho-ERK & HIF-1 $\alpha$ \\
\hline Lymph node & 1 & & & & & & & & \\
\hline Pathological grade & & 1 & & & & & & & \\
\hline Extension & & & 1 & & & & & & \\
\hline Tumour size & 0.11 & 0.22 & 0.17 & 1 & & & & & \\
\hline EGFR & & 0.16 & & & 1 & & & & \\
\hline SIAH & & 0.11 & & & & 1 & & & \\
\hline Ki67 & & & & & & 0.76 & 1 & & \\
\hline Phospho-ERK & & -0.10 & & & 0.14 & & & 1 & \\
\hline HIF- $1 \alpha$ & -0.14 & 0.22 & -0.13 & & & 0.12 & & & 1 \\
\hline
\end{tabular}

making SIAH a useful and tumour-specific biomarker in pancreatic cancer.

The IHC staining of four signalling components in the K-RAS pathway. Tumour biospecimens were stained with anti-EGFR, anti-HER2, anti-phospho-ERK and anti-SIAH antibodies (Figure 1C and D, Table 2 and data not shown). The membrane receptors, EGFR and HER2 were evaluated by both extent and intensity of staining on a scale of $0-4$ for their heterogeneous expression (Table 2). EGFR expression is summarised in Figure 1C and $\mathrm{D}$ and Table 2. Most tumour samples did not show appreciable HER2 expression (Table 2). Thus, HER2 was excluded in our statistical analyses. Phospho-ERK staining was scored by staining percentage on a scale of $0-100 \%$ positivity. Phospho-ERK expression was observed in the nuclei of tumour cells as well as some adjacent tumour stroma. SIAH staining was scored by staining percentage on a scale of $0-100 \%$ positivity (Figure $1 \mathrm{C}$ and $\mathrm{D}$ and Table 2).

For our pancreatic cancer patients, SIAH expression was detected in 100\%, EGFR expression was detected in $85 \%$ and phospho-ERK expression was detected in $81 \%$ of them (Table 2). In their resected tumours, SIAH, EGFR and phospho-ERK together marked $98 \%$ of all neoplastic cells. In 234 TMA tumour cores that retained high-quality IHC staining of EGFR, phosphoERK and SIAH, SIAH marked 79\%, EGFR marked $70 \%$ and phospho-ERK marked 62\% of the tumour cells (Figure 1C and Table 2). The results show that these three signalling components in the K-RAS pathway are pertinent biomarkers in pancreatic cancer.

Increased expression of HIF- $1 \alpha$ is associated with shortened survival post surgery. HIF- $1 \alpha$ expression was examined to assess the hypoxic response in pancreatic tumours. HIF- $1 \alpha$ staining was scored by staining percentage on a scale of 2-90\% (Figure 2D and Table 2). HIF- $1 \alpha$ expression was quite heterogeneous. The greatest level of expression was concentrated in the nucleus of pancreatic cancer cells; however, low cytoplasmic expression levels were also detected. Representative HIF- $1 \alpha$ staining in patients with distinct survival rates was shown (Figure 2D). The median expression level of HIF- $1 \alpha$ was $40 \%$. Patients expressing HIF- $1 \alpha$ above $40 \%$ had a median survival time of 14.8 months, whereas patients expressing HIF- $1 \alpha$ below $40 \%$ had a median survival time of 19.2 months with $P=0.004$ (Figure 2C). Only $13.3 \%$ of patients with high HIF- $1 \alpha$ expression survived 3 years or longer. Patients with HIF- $1 \alpha$ below median $40 \%$ expression showed a statistically significant increase in 5-year survival compared with the patients with high HIF- $1 \alpha$ expression (Figure 2C and D).

The IHC staining of Ki67 in resectable pancreatic cancer. The tumour proliferation index was measured and Ki67 staining was scored by percentage on a scale of $5-90 \%$ positivity (Figure $2 \mathrm{~F}$ and Table 2). Only one patient did not display detectable Ki67 staining. $\mathrm{Ki} 67$ is expressed in tumour/cancerous cells; tumour stroma and immune cells showed no Ki67-positive staining. Ki67 expression increased with pathological grades (Figure $2 \mathrm{~F}$ ), marking proliferating tumour cells in pancreatic cancer. The median expression level of Ki67 was 40\%. The median survival was 14.6 months for patients expressing Ki67 above $40 \%$ but 18.7 months for patients expressing Ki67 below 40\% (Figure 2E and F). Only 15.3\% of patients with high Ki67 expression survived 3 years or longer, vs a $28.6 \%$ survival rate for patients with low Ki67 expression; however, the results were not statistically significant $(P=0.146$; Figure $2 \mathrm{E})$.

A combination of four clinicopathologic predictors and five biomarkers is associated with improved accuracy to predict patient survival post surgery. For clinical variables prognostic in pancreatic cancer, univariate analysis showed that tumour grade $(P<0.001)$ was a significant prognostic predictor of patient survival; the Kaplan-Meier survival curves indicated increased tumour grade associating with poor survival (Figure 2A). Other clinical variables including lymph node status, tumour extension beyond the pancreas, UICC clinical stage, margin status, perineural invasion, angiolymphatic invasion or adjuvant therapy were not significant prognostic factors of patient survival (Table 1). 
A

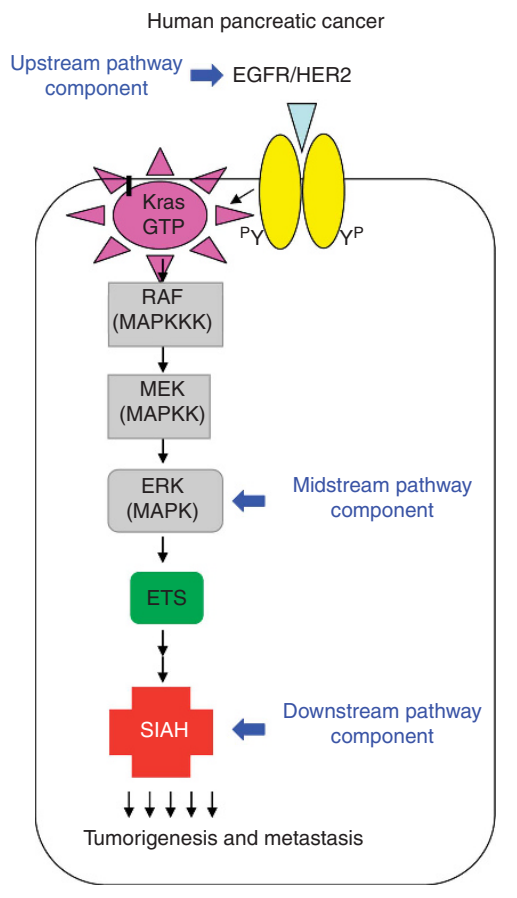

B

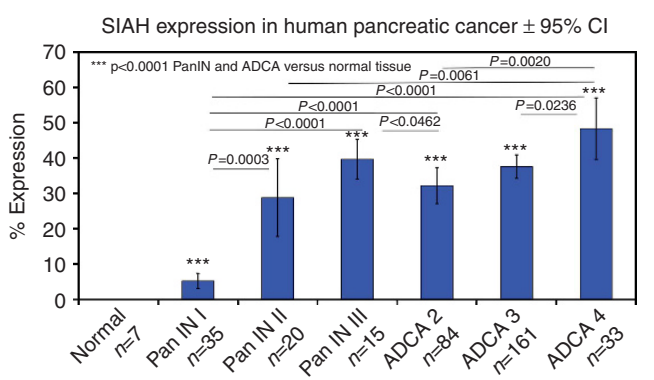

C

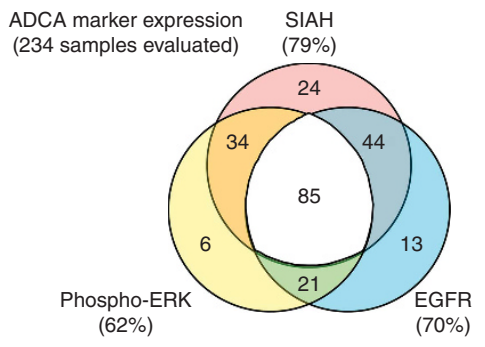

D

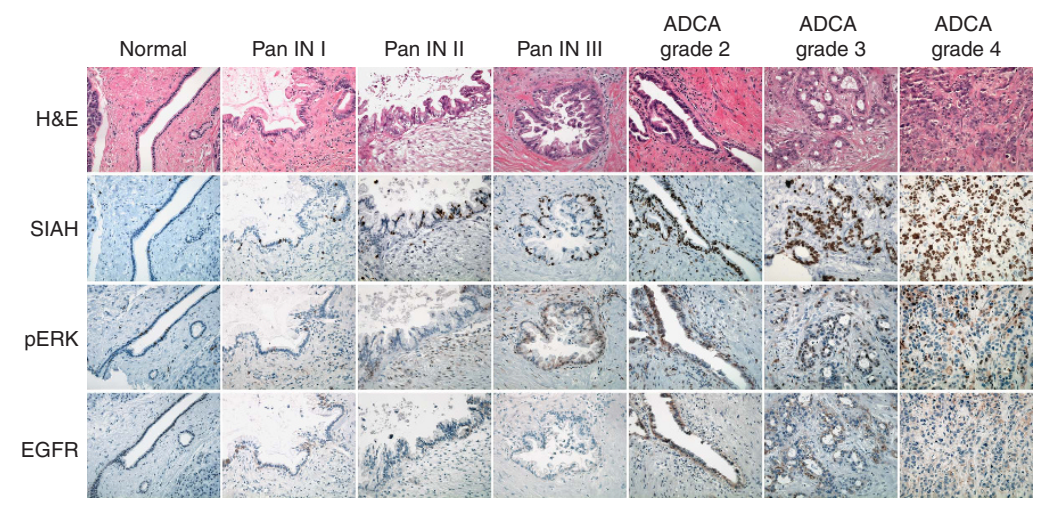

Figure 1. EGFR, phospho-ERK and SIAH expression in resectable pancreatic cancer. (A) A schematic illustration of the oncogenic K-RAS pathway is shown. The expression of the upstream receptors, EGFR/HER2, the midstream kinase, ERK and the most downstream E3 ligase, SIAH, were examined. (B) SIAH expression is upregulated as human pancreatic cancer progresses to advanced stages. Percentage of SIAH ( + ) cells increased progressively through well-defined stages: PanIN-1, PanIN-2, PanIN-3 to adenocarcinoma (ADCA 2, ADCA 3 and ADCA 4). Student's t-tests were performed and $P$-values less than 0.05 were considered statistically significant as shown. (C) Venn diagram representation of single, double and triple staining of SIAH, phospho-ERK and EGFR expression on 234 tumour cores with a complete and overlapping set of the IHC staining, was shown. (D) Representative EGFR, phospho-ERK and SIAH staining in PanIN and ADCA stages are shown. SIAH marked tumour cells specifically. Note the lack of SIAH staining in normal pancreas and tumour stroma.

Univariate analysis with the Cox proportional hazards model showed that HIF- $1 \alpha$ expression has increased power to predict survival. HIF- $1 \alpha$ expression was a significant predictor of survival $(P=0.005)$ with a hazard ratio of $1.19(95 \% \mathrm{CI}: 1.06-1.35)$ for a unit of $20 \%$ increment, indicating that a higher percentage of HIF- $1 \alpha$ expression is associated with reduced survival (Table 1 and a $P$-value from a log-rank test for binary HIF- $1 \alpha$ expression less than or equal to 40 as shown in Figure 2C). None of four biomarkers (EGFR, SIAH, phospho-ERK or Ki67) were significant predictors of survival in pancreatic cancer individually (Table 1). The analysis data set varies slightly for each biomarker because of minor TMA imperfections (Table 2). HIF-1 $\alpha$ expression correlated with tumour grade (Spearman's rank correlation coefficient of $0.22, P=0.025$ ), but was not significantly correlated with other clinicopathological predictors (Table 3). Ki67 expression was highly correlated with SIAH (Spearman's rank correlation coefficient of $0.76, P<0.001$ ), but was not correlated significantly with other clinical variables (Table 3). The only statistically significant correlation was between tumour grade and tumour size (Spearman's rank correlation coefficient of $0.22, P=0.008$; Table 3).

To determine whether the addition of these five molecular biomarkers can improve prognostic accuracy after adjusting for clinicopathological variables, we performed multiple survival analyses using the Cox $\mathrm{PH}$ regression models. After adjusting for lymph node status, pathologic grade, tumour extension beyond the pancreas and tumour size, none of the signalling components of the K-RAS pathway (EGFR, phospho-ERK and SIAH) were statistically significant predictors of patient survival (Table 4). Pathological grade remained a significant prognostic factor in the Cox PH regression model. Ki67 and HIF- $1 \alpha$ expression trended but did not reach statistical significance $(P=0.247$ and $P=0.149$, respectively; Table 4). One possible explanation for the disappearance of significance of HIF- $1 \alpha$ expression from univariate analysis to multivariate analysis may be that HIF- $1 \alpha$ was significantly correlated with pathological tumour grade. In further exploratory analysis, HIF- $1 \alpha$ expression became statistically significant $(P=0.004)$ once pathological tumour grade was intentionally removed.

Survival ROC curves. Consistent with previous reports, clinical variables, such as tumour size and pathologic grade, are linked to survival in pancreatic cancer (Tables 1 and 4). Our biomarker analysis revealed that HIF- $1 \alpha$ expression may be associated with reduced survival in pancreatic cancer. Here, we proposed five models for predicting patient survival: (A) five biomarkers of Ki67, HIF-1 $\alpha$, EGFR, phospho-ERK and SIAH, (B) clinicopathologic only, (C) clinicopathologic variables plus Ki67, (D) clinicopathologic variables plus HIF- $1 \alpha$, (E) clinicopathologic variables plus the five biomarkers. To evaluate predictive accuracy of these models, time-dependent survival ROC curves were constructed for 3-year survival predictions (Figure $3 \mathrm{~A}$ ). Area under the curve (AUC) for 3 -year survival was used to numerically compare the performance of these five models. The AUC increased from 0.685 for five biomarkers only (Model A) to 0.751 for the clinicopathologic variables alone (Model B), and to 0.773 with the addition of Ki67 (Model C), and to 0.788 with the addition of HIF-1 $\alpha$ (Model D), respectively (Figure $3 \mathrm{~B}$ ). The AUC combining the four clinicopathological variables plus the five molecular biomarkers together (Model E) was 0.850 (Figure 3B).

A time-dependent $\operatorname{AUC}(t)$ curve for continuous time points was also plotted to investigate the predictive accuracy of the five models with survival. The data suggest that the absent or decreased HIF-1 $\alpha$ 
A

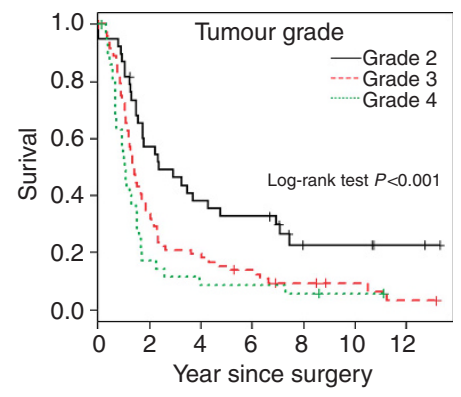

$\begin{array}{llllllll}\text { Grade } 2 \text { (Pt no.) } & 38 & 21 & 14 & 12 & 5 & 5 & 2\end{array}$

$\begin{array}{llllllll}\text { Grade } 3 \text { (Pt no.) } & 73 & 24 & 14 & 9 & 5 & 3 & 1\end{array}$

$\begin{array}{llllllll}\text { Grade } 4 \text { (Pt no.) } & 36 & 6 & 3 & 3 & 2 & 1 & 0\end{array}$

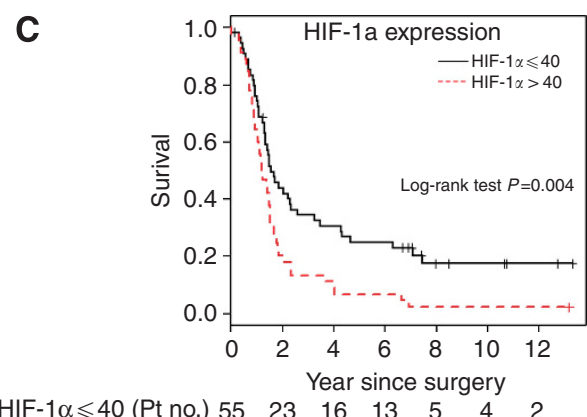

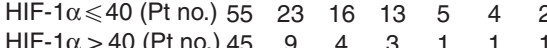

E

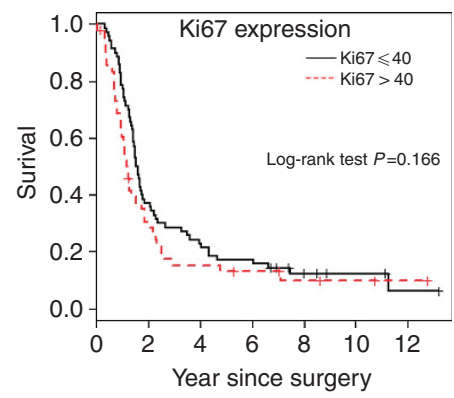

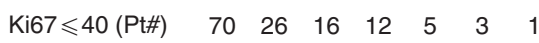

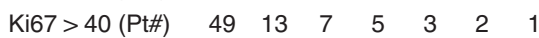

B

$\mathrm{SIAH}$

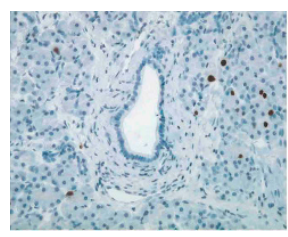

Normal

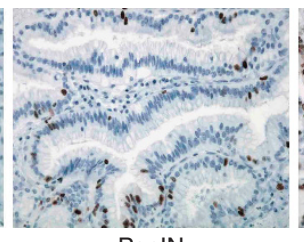

PanIN

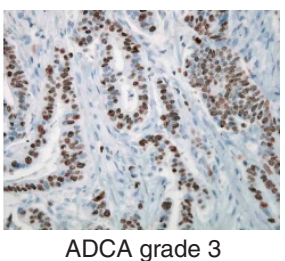

Tissue microarray - grade 3 ADCA
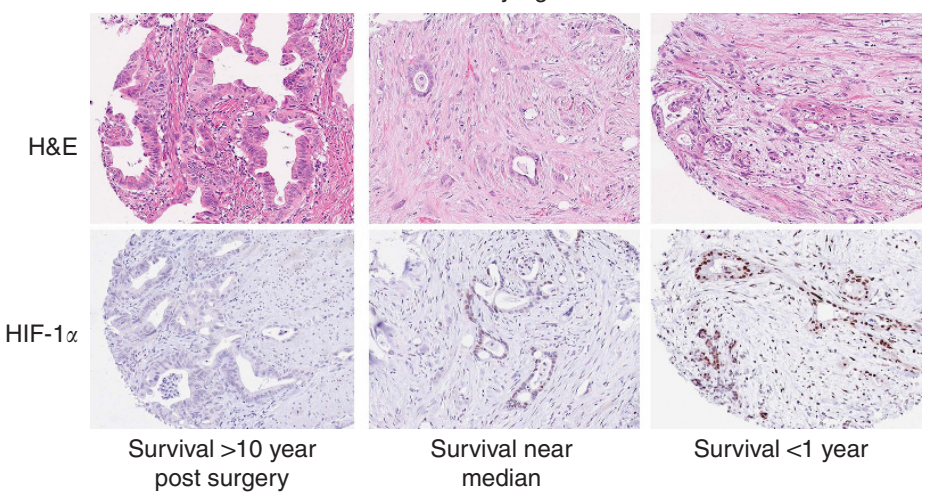

$\mathbf{F}$

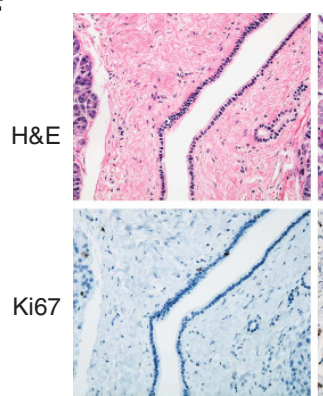

Normal

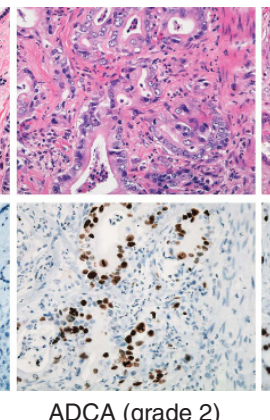

ADCA (grade 2)

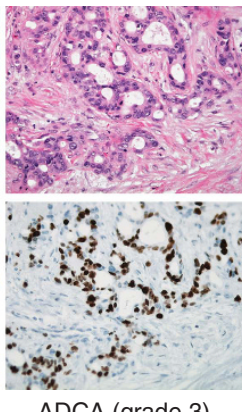

ADCA (grade 3)

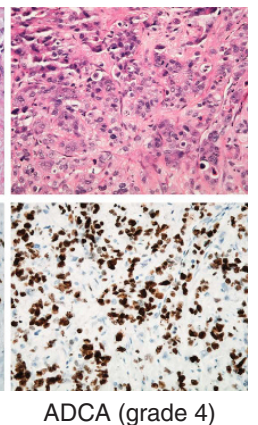

Figure 2. Increased tumour grades and heightened hypoxia response predicts poor prognosis and adverse outcome in resectable pancreatic cancer. Kaplan-Meier curves are used to predict patient survival with the number of patients at risk each year during the 12 years of follow-up indicated (A, C and E). Representative IHC images are shown (B, D and F). Haematoxylin and eosin (H\&E) staining is shown to view the tissue histology. (A) Kaplan-Meier curve shows that tumour grade is a significant predictor of patient survival $(P<0.001)$. By univariate analysis, a higher tumour grade is associated with worse prognosis. (B) Representative SIAH expression is shown in normal pancreas, PanINs and ADCAs. (C) The Kaplan-Meier curve shows that increased HIF-1 $\alpha$ expression is correlated with reduced survival in pancreatic cancer. (D) The representative HIF- $1 \alpha$ staining is shown in Grade 3 ADCA. These patients have similar clinicopathological diagnoses but they experienced markedly different outcomes and survival. Increased HIF-1 $\alpha$ expression appears to be correlated with reduced survival in pancreatic cancer. (E) The Kaplan-Meier curves for patients with Ki67 staining above and below the median expression level (40\%) were shown. (F) Representative images of Ki67 staining in normal pancreas and ADCA 2, 3 and 4 are shown. Increased Ki67 expression is associated with advanced stages of ADCAs.

is associated with a good prognosis for long-term survival; and the combination of the five molecular biomarkers (EGFR, phosphoERK, SIAH, Ki67 and HIF- $1 \alpha$ ) in the K-RAS/HIF- $1 \alpha$ pathway and the four clinicopathologic variables (tumour size, pathological grade, margin and lymph node status) can improve prognostic accuracy of patient survival post surgery (Figure $3 \mathrm{~B}$ ).

\section{DISCUSSION}

Ninety-five percent mortality rates in pancreatic cancer have shown little improvement despite intense research efforts over 40 years. Patient prognosis appears to be influenced in large part by pancreatic tumour genome biology and cancer cell dissemination rather than by adequate resection (Wolfgang et al, 2013). Favourable clinicopathological prognostic indicators include small tumour size, negative nodal status, negative resection margin and well-differentiated carcinomas. Evaluation of numerous molecular biomarkers as prognostic factors has achieved inconsistent results (Sawyers, 2008; Costello et al, 2012; Winter et al, 2013). Discovery and validation of logical and useful biomarkers to improve prognosis and guide effective therapies is important to combat pancreatic cancer. Here, we report that SIAH is a tumour-specific biomarker in pancreatic cancer (Figure 1); HIF-1 $\alpha$ shows some promise as a prognostic variable (Figure 2); and individually, the selected five biomarkers, EGFR, phospho-ERK, SIAH, Ki67 and HIF-1 $\alpha$ are weakly prognostic or not at all, but when combined them with the four clinicopathological predictors (tumour size, pathological grade, margin status 
and lymph node status) together, it yields increased prognostic accuracy in resectable pancreatic cancer (Figure 3). One important caveat is that only resected pancreatic tumours were examined in this study. As $\sim 85 \%$ of pancreatic cancer patients have incurable diseases without surgical options, we do not know whether the K-RAS/Ki67/HIF- $1 \alpha$ pathway biomarkers will synergise with clinicopathologic predictors in inoperable pancreatic cancers. With rapid autopsy programmes in pancreatic cancer, we hope to analyse and validate our findings on HIF- $1 \alpha$, EGFR, phospho-ERK, SIAH and Ki67 alone and in combination with clinical parameters in the context of systemic metastasis in the future.

We have demonstrated that increased HIF- $1 \alpha$ expression correlates with increasing pathological grades, consistent with previous findings in pancreatic cancer (Kitada et al, 2003; Shibaji et al, 2003; Guillaumond et al, 2013). HIF- $1 \alpha$ overexpression is a negative prognostic variable in several human cancers (Semenza, 2003; Wilson and Hay, 2011). Here, we demonstrate that increased HIF- $1 \alpha$ expression correlates with increasing pathological grades and poor prognosis, and HIF- $1 \alpha$ is overexpressed in $69 \%$ of pancreatic tumour specimens in this study within 147 patients, consistent with the results of two prior, small-scale studies (Zhong et al, 1999; Kitada et al, 2003). In the first study, HIF-1 $\alpha$ was found to be overexpressed in four out of five primary pancreatic cancer specimens (Zhong et al, 1999). In the second study, overexpression of HIF- $1 \alpha$ was detected in $59.2 \%$ (29 out of 49 cases) of pancreatic cancer specimens (Kitada et al, 2003). Previous studies have found correlations between HIF- $1 \alpha$ expression and larger tumour size, advanced UICC stage, and metastatic disease in pancreatic cancer (Kitada et al, 2003; Shibaji et al, 2003; Chang et al, 2011). Our results have shown that high HIF- $1 \alpha$ expression levels in pancreatic adenocarcinomas-with staining percentages above $40 \%$ - signify a notable decrease in patient survival. Furthermore, survival ROC analysis suggests that adding HIF- $1 \alpha$ expression alone to the list of existing clinicopathologic parameters will strengthen clinical prediction of survival in pancreatic cancer (AUC 0.751 vs 0.788 ; Figure 3). The data suggest that decreased Ki67 may have a good prognosis for short-term survival, within a year or so, whereas the lack of or the decreased HIF- $1 \alpha$ may have a good prognosis for long-term survival. The different onset and distinct dynamics of Ki67 and HIF-1 $\alpha$ expression patterns may reflect the altered biology and successful adaptation of pancreatic cancer cells to the

Table 4. Cox PH regression models with four clinical variables and five biomarkers

\begin{tabular}{|c|c|c|c|c|c|}
\hline Predictors & Level & Hazard ratio & $95 \% \mathrm{Cl}$ & $P$-value & \\
\hline Lymph node positive & $\begin{array}{l}\text { No } \\
\text { Yes }\end{array}$ & $\begin{array}{l}1.000 \\
0.915\end{array}$ & $\overline{0.536}$ & $\overline{1.560}$ & $\overline{0.745}$ \\
\hline Pathological grade & $\begin{array}{l}2 \\
3 \\
4\end{array}$ & $\begin{array}{l}1.000 \\
2.665 \\
3.944\end{array}$ & $\begin{array}{c}-\overline{1} \\
1.329 \\
1.736\end{array}$ & $\begin{array}{l}-\overline{340} \\
8.960\end{array}$ & $\begin{array}{c}\overline{-} \\
0.006 \\
0.001\end{array}$ \\
\hline Extension & $\begin{array}{l}\text { No } \\
\text { Yes }\end{array}$ & $\begin{array}{l}1.000 \\
1.280\end{array}$ & $\overline{0.748}$ & $2 . \overline{190}$ & $\overline{0.368}$ \\
\hline Tumour size & & 1.180 & 0.907 & 1.530 & 0.219 \\
\hline EGFR & & 1.056 & 0.895 & 1.250 & 0.519 \\
\hline SIAH & & 0.998 & 0.980 & 1.020 & 0.822 \\
\hline Ki67 & & 1.010 & 0.993 & 1.030 & 0.247 \\
\hline Phospho-ERK & & 0.997 & 0.989 & 1.000 & 0.443 \\
\hline HIF- $1 \alpha$ & & 1.005 & 0.998 & 1.010 & 0.149 \\
\hline
\end{tabular}

A Time-dependent survival ROCs at 3 years

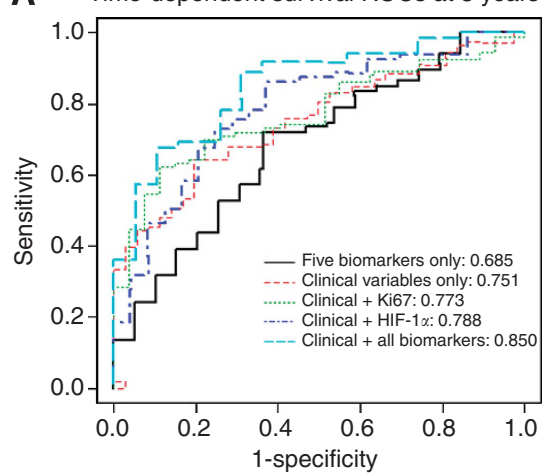

B Area under curve plots for the first 5 years

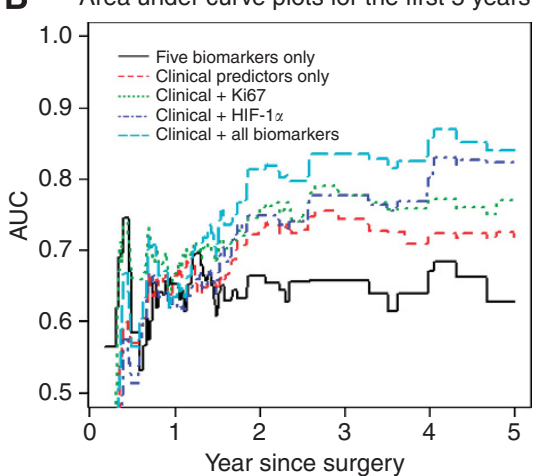

Figure 3. A combination of four clinical markers and five biomarkers has more accurate prognostic value in predicting 3- or 5-year patient survival in resectable pancreatic cancer. (A) Three-year survival prediction: time-dependent survival ROCs at 3 years based on five predictive models are shown. Five biomarkers only (black line) and clinicopathological predictors only (red dotted line) served as the controls. Ki67 in combination with the clinical predictors (green dotted line) have improved predictive power, as with HIF-1 $\alpha$ in combination with the clinical predictors (blue dotted line). When all five biomarkers are combined with the clinical predictors together (aqua/cyan dotted line), there is a significant improvement in predicting patient survival post-pancreatic surgeries. (B) Five-year survival prediction: AUC(t) plots for the first 5 years based on five predictive models are shown (black line). Five biomarkers only (black line) and clinicopathological predictors only (red dotted line) served as the controls. Ki67 in combination with the clinical predictors (green dotted line) have an improved predictive power, as with HIF- $1 \alpha$ (blue dotted line). When all five biomarkers are combined with the four clinical predictors together (aqua/cyan dotted line), there is a significant improvement in predictive power based on AUC analysis. 
local environments during the course of aggressive tumour growth and metastasis in vivo. Taken together, our results support the notion that HIF- $1 \alpha$ expression is a useful prognostic indictor of adverse clinical outcome in resectable pancreatic cancer.

Ki67 was widely expressed in pancreatic tumours (95\%) evaluated in this study. Two previous studies have investigated the role of $\mathrm{Ki} 67$ as a prognostic marker in pancreatic adenocarcinomas with negative results (Stanton et al, 2003; Lebe et al, 2004) using tumour specimens taken from fewer than 60 patients. In our larger study $(n=147)$, Kaplan-Meier survival analysis did not reveal a statistically significant difference in 5 -year survival between patients with low and high levels of Ki67 expression $(P=0.166)$, consistent with the previous reports (Figure 2E).

Through multivariate analysis, we found that a significant correlation exists between the overexpression of HIF- $1 \alpha$ with decreased postoperative survival. Besides HIF- $1 \alpha$, the addition of Ki67 and the three additional biomarkers, EGFR, phospho-ERK and SIAH, in the oncogenic K-RAS signalling pathway (the upstream, midstream and downstream signalling modules, respectively) can further increase the accuracy of a prognosis of patient survival. Hyperactive K-RAS signalling is a major menace in human pancreatic cancer. Currently, oncogenic K-RAS has remained an 'undruggable' target in cancer. Additional studies to validate the prognostic values of the combination of clinicopathological predictors, and these RAS pathway biomarkers may assist to predict patient survival after surgical resection in the future.

\section{ACKNOWLEDGEMENTS}

We are indebted to Drs Edward Leof, Stephen Deutsch, Edward Johnson, Frank Lattanzio, Roger Perry, Richard Hoefer, Vasilena Zheleva, Mr John Crooks and Ms Lauren Siewertsz van Reesema for their critical reading of this manuscript and invaluable critiques. RLS was supported by the Mayo Clinic Pobanz Family Predoctoral Research Fellowship. This work is supported by 2010 AACR-PanCAN Innovative Grant (AACR-PanCan no. 169458), 2005 Lustgarten Foundation for Pancreatic Cancer Research (RFA05-046), National Institute of General Medical Sciences (GM069922-06S1), National Cancer Institute (CA140550) and the Mayo Pancreatic Cancer SPORE Pilot Grant Award to AHT.

\section{CONFLICT OF INTEREST}

The authors declare no conflict of interest.

\section{AUTHOR CONTRIBUTION}

RQ conducted biostatistical analyses. TCS identified representative tumour paraffin blocks, guided pancreatic TMA construction and controlled the quality of the IHC staining and scoring. NRR reviewed patient charts, extracted clinical data from the Mayo Clinic tumour registry, and established the database. RLS assisted with patient de-identification, data entry, data processing and IHC image capture. TS assisted with the clinical data extraction, STC recruited and identified pancreatic cancer patients for clinical studies. GMP oversaw the Mayo Clinic Pancreatic SPORE operations and provided the TMA slides and scientific guidance to this study. AHT designed the experiments, supervised this project, wrote the manuscript and generated the figures and tables. All authors discussed the results and contributed to the extensive revisions of this manuscript.

\section{REFERENCES}

Ahmed AU, Schmidt RL, Park CH, Reed NR, Hesse SE, Thomas CF, Molina JR, Deschamps C, Yang P, Aubry MC, Tang AH (2008) Effect of disrupting seven-in-absentia homolog 2 function on lung cancer cell growth. J Natl Cancer Inst 100(22): 1606-1629.

Akakura N, Kobayashi M, Horiuchi I, Suzuki A, Wang J, Chen J, Niizeki H, Kawamura K, Hosokawa M, Asaka M (2001) Constitutive expression of hypoxia-inducible factor-1alpha renders pancreatic cancer cells resistant to apoptosis induced by hypoxia and nutrient deprivation. Cancer Res 61(17): 6548-6554.

Ardito CM, Gruner BM, Takeuchi KK, Lubeseder-Martellato C, Teichmann N, Mazur PK, Delgiorno KE, Carpenter ES, Halbrook CJ, Hall JC, Pal D, Briel T, Herner A, Trajkovic-Arsic M, Sipos B, Liou GY, Storz P, Murray NR, Threadgill DW, Sibilia M, Washington MK, Wilson CL, Schmid RM, Raines EW, Crawford HC, Siveke JT (2012) EGF receptor is required for KRAS-induced pancreatic tumorigenesis. Cancer Cell 22(3): 304-317.

Chang Q, Jurisica I, Do T, Hedley DW (2011) Hypoxia predicts aggressive growth and spontaneous metastasis formation from orthotopically grown primary xenografts of human pancreatic cancer. Cancer Res 71(8): 3110-3120.

Conroy T, Desseigne F, Ychou M, Bouche O, Guimbaud R, Becouarn Y, Adenis A, Raoul JL, Gourgou-Bourgade S, de la Fouchardiere C, Bennouna J, Bachet JB, Khemissa-Akouz F, Pere-Verge D, Delbaldo C, Assenat E, Chauffert B, Michel P, Montoto-Grillot C, Ducreux M (2011) FOLFIRINOX versus gemcitabine for metastatic pancreatic cancer. N Engl J Med 364(19): 1817-1825.

Costello E, Greenhalf W, Neoptolemos JP (2012) New biomarkers and targets in pancreatic cancer and their application to treatment. Nat Rev Gastroenterol Hepatol 9(8): 435-444.

Guillaumond F, Leca J, Olivares O, Lavaut MN, Vidal N, Berthezene P, Dusetti NJ, Loncle C, Calvo E, Turrini O, Iovanna JL, Tomasini R, Vasseur S (2013) Strengthened glycolysis under hypoxia supports tumor symbiosis and hexosamine biosynthesis in pancreatic adenocarcinoma. Proc Natl Acad Sci USA 110(10): 3919-3924.

Heagerty PJ, Zheng Y (2005) Survival model predictive accuracy and ROC curves. Biometrics 61(1): 92-105.

Hidalgo M (2010) Pancreatic cancer. N Engl J Med 362(17): 1605-1617. Hruban RH, Goggins M, Parsons J, Kern SE (2000) Progression model for pancreatic cancer. Clin Cancer Res 6(8): 2969-2972.

Javle MM, Gibbs JF, Iwata KK, Pak Y, Rutledge P, Yu J, Black JD, Tan D, Khoury T (2007) Epithelial-mesenchymal transition (EMT) and activated extracellular signal-regulated kinase (p-Erk) in surgically resected pancreatic cancer. Ann Surg Oncol 14(12): 3527-3533.

Katz MH, Wang H, Fleming JB, Sun CC, Hwang RF, Wolff RA, Varadhachary G, Abbruzzese JL, Crane CH, Krishnan S, Vauthey JN, Abdalla EK, Lee JE, Pisters PW, Evans DB (2009) Long-term survival after multidisciplinary management of resected pancreatic adenocarcinoma. Ann Surg Oncol 16(4): 836-847.

Kitada T, Seki S, Sakaguchi H, Sawada T, Hirakawa K, Wakasa K (2003) Clinicopathological significance of hypoxia-inducible factor-1alpha expression in human pancreatic carcinoma. Histopathology 43(6): $550-555$.

Lebe B, Sagol O, Ulukus C, Coker A, Karademir S, Astarcioglu H, Kupelioglu A, Astarcioglu I, Obuz F (2004) The importance of cyclin D1 and Ki67 expression on the biological behavior of pancreatic adenocarcinomas. Pathol Res Pract 200(5): 389-396.

Maitra A, Hruban RH (2008) Pancreatic cancer. Annu Rev Pathol 3: 157-188. Sawyers CL (2008) The cancer biomarker problem. Nature 452(7187): 548-552.

Schmidt RL, Park CH, Ahmed AU, Gundelach JH, Reed NR, Cheng S, Knudsen BE, Tang AH (2007) Inhibition of RAS-mediated transformation and tumorigenesis by targeting the downstream E3 ubiquitin ligase seven in absentia homologue. Cancer Res 67(24): 11798-11810.

Sebolt-Leopold JS, Herrera R (2004) Targeting the mitogen-activated protein kinase cascade to treat cancer. Nat Rev Cancer 4(12): 937-947.

Semenza GL (2003) Targeting HIF-1 for cancer therapy. Nat Rev Cancer 3(10): 721-732.

Shibaji T, Nagao M, Ikeda N, Kanehiro H, Hisanaga M, Ko S, Fukumoto A, Nakajima Y (2003) Prognostic significance of HIF-1 alpha overexpression in human pancreatic cancer. Anticancer Res 23(6C): 4721-4727. 
Siegel R, Naishadham D, Jemal A (2013) Cancer statistics, 2013. CA Cancer J Clin 63(1): 11-30.

Sohn TA, Yeo CJ, Cameron JL, Koniaris L, Kaushal S, Abrams RA, Sauter PK, Coleman J, Hruban RH, Lillemoe KD (2000) Resected adenocarcinoma of the pancreas-616 patients: results, outcomes, and prognostic indicators. J Gastrointest Surg 4(6): 567-579.

Spivak-Kroizman TR, Hostetter G, Posner R, Aziz M, Hu C, Demeure MJ, Von Hoff D, Hingorani SR, Palculict TB, Izzo J, Kiriakova GM, Abdelmelek M, Bartholomeusz G, James BP, Powis G (2013) Hypoxia triggers hedgehog-mediated tumor-stromal interactions in pancreatic cancer. Cancer Res 73(11): 3235-3247.

Stanton KJ, Sidner RA, Miller GA, Cummings OW, Schmidt CM, Howard TJ, Wiebke EA (2003) Analysis of Ki-67 antigen expression, DNA proliferative fraction, and survival in resected cancer of the pancreas. Am J Surg 186(5): 486-492.

Valle JW, Palmer D, Jackson R, Cox T, Neoptolemos JP, Ghaneh P, Rawcliffe CL, Bassi C, Stocken DD, Cunningham D, O’Reilly D, Goldstein D, Robinson BA, Karapetis C, Scarfe A, Lacaine F, Sand J, Izbicki JR, Mayerle J, Dervenis C, Olah A, Butturini G, Lind PA, Middleton MR, Anthoney A, Sumpter K, Carter R, Buchler MW (2014) Optimal duration and timing of adjuvant chemotherapy after definitive surgery for ductal adenocarcinoma of the pancreas: ongoing lessons from the ESPAC-3 study. J Clin Oncol 32(6): 504-512.
Vogelstein B, Papadopoulos N, Velculescu VE, Zhou S, Diaz Jr LA, Kinzler KW (2013) Cancer genome landscapes. Science 339(6127): 1546-1558.

Von Hoff DD, Korn R, Mousses S (2009) Pancreatic cancer-could it be that simple? A different context of vulnerability. Cancer Cell 16(1): 7-8.

Wilson WR, Hay MP (2011) Targeting hypoxia in cancer therapy. Nat Rev Cancer 11(6): 393-410.

Winter JM, Yeo CJ, Brody JR (2013) Diagnostic, prognostic, and predictive biomarkers in pancreatic cancer. J Surg Oncol 107(1): 15-22.

Wolfgang CL, Herman JM, Laheru DA, Klein AP, Erdek MA, Fishman EK, Hruban RH (2013) Recent progress in pancreatic cancer. CA Cancer J Clin 63(5): 318-348.

Zhong H, De Marzo AM, Laughner E, Lim M, Hilton DA, Zagzag D, Buechler P, Isaacs WB, Semenza GL, Simons JW (1999) Overexpression of hypoxia-inducible factor 1alpha in common human cancers and their metastases. Cancer Res 59(22): 5830-5835.

This work is published under the standard license to publish agreement. After 12 months the work will become freely available and the license terms will switch to a Creative Commons AttributionNonCommercial-Share Alike 4.0 Unported License. 